\title{
Hidden Coexisting Attractors in a Fractional-Order System without Equilibrium: Analysis, Circuit Implementation, and Finite-Time Synchronization
}

\author{
Guangchao Zheng $\mathbb{D}^{1,2,3}$ Ling Liu, ${ }^{1,2}$ and Chongxin Liu ${ }^{1,2}$ \\ ${ }^{1}$ State Key Laboratory of Electrical Insulation and Power Equipment, Xian Jiaotong University, Xian 710049, China \\ ${ }^{2}$ School of Electrical Engineering, Xian Jiaotong University, Xian 710049, China \\ ${ }^{3}$ Baoding Power Supply Branch of State Grid Hebei Electric Power Supply Co. Ltd., Baoding 071000, China \\ Correspondence should be addressed to Guangchao Zheng; zhenggc16@foxmail.com
}

Received 24 February 2019; Revised 5 June 2019; Accepted 3 July 2019; Published 22 July 2019

Academic Editor: Ivo Petras

Copyright ( 2019 Guangchao Zheng et al. This is an open access article distributed under the Creative Commons Attribution License, which permits unrestricted use, distribution, and reproduction in any medium, provided the original work is properly cited.

In this paper, a novel three-dimensional fractional-order chaotic system without equilibrium, which can present symmetric hidden coexisting chaotic attractors, is proposed. Dynamical characteristics of the fractional-order system are analyzed fully through numerical simulations, mainly including finite-time local Lyapunov exponents, bifurcation diagram, and the basins of attraction. In particular, the system can generate diverse coexisting attractors varying with different orders, which presents ample and complex dynamic characteristics. And there is great potential for secure communication. Then electronic circuit of the fractional-order system is designed to help verify its effectiveness. What is more, taking the disturbances into account, a finite-time synchronization of the fractional-order chaotic system without equilibrium is achieved and the improved controller is proven strictly by applying finite-time stable theorem. Eventually, simulation results verify the validity and rapidness of the proposed method. Therefore, the fractional-order chaotic system with hidden attractors can present better performance for practical applications, such as secure communication and image encryption, which deserve further investigation.

\section{Introduction}

Since Lorenz system [1] was put forward in the 1960s, chaos theory has made a great leap forward and researchers have never ceased to delve deeply into it. Among them, threedimensional autonomous chaotic systems are investigated most extensively at home and abroad, such as Rössler system [2], Chen system [3], Lü system [4], and Liu system [5]. And these chaotic systems have achieved comprehensive application in real life, including secure communication, image encryption, medicine, and aerospace.

In recent years, however, increasing attention has been paid to hidden attractors, which were discovered first in the Chua'circuit [6]. For those conventional chaotic systems above, their attractors are called self-excited attractors [7], whose basins of attraction can intersect unstable equilibrium points. In contrast, the attractors are defined as hidden attractors if its basins of attraction do not touch any unstable equilibrium point. The current literatures indicate that hidden attractors originate from several types of particular dynamical systems. And to be specific, the dynamical systems with one stable equilibrium $[8,9]$, a line equilibrium $[10,11]$, or no equilibrium [12-15] can all fall into this category. Even there are hidden attractors found in some special chaotic systems [16] which have both unstable equilibrium and stable equilibria. It is the presence of hidden attractors that pose a new challenge to Shilnikov criteria which illustrate that no less than one unstable equilibrium is needed to generate chaos. Meanwhile, with the lack of correlation between hidden attractors and equilibria, precise localization of these unpredictable attractors can be of great intractability. In addition, it also has been a focus of concern about coexistence 
of hidden attractors [17-20], which presents more complex and diverse dynamic behavior, and enhances the security ability in the field of cryptography application.

However, the existing research findings about hidden attractors have been mostly focused on integer-order dynamical systems and relatively few efforts have been done for fractional-order systems with hidden attractors [16, 21-24]. The latter are worthy of further investigation owing to excellent characteristics of fractional calculus. To begin with, fractional-order systems, a further generalization, are more applicable by contrast with integer-order systems. Indeed, it can be said integer-order systems are the particular case of fractional-order systems and the idealization of real matter. Accordingly, fractional-order systems can describe the physical characteristics of the system engineering more clearly and reflect the essence and law of things in application domain. Then, fractional-order systems have special historical memory function, which are suitable for describing systems that rely on historical information. Furthermore, they can exhibit more complex and richer dynamic characteristics, with parameter selection more flexible. Thus, fractional calculus shows strong potential in practical engineering applications [25-28].

Based on the above issues, this paper discusses the fractional-order form of a new 3D symmetric system without equilibrium and gives emphasis to the influence of the order on dynamic behavior of the system. The detailed dynamic analysis was carried out, mainly including existence of multiple attractors. And a finite-time synchronization of the fractional-order chaotic system with hidden attractors is realized. It has enormous value for the application of chaos technology in practical engineering field, especially in secure communication.

This paper is organized as follows. In Section 2, several definitions of fractional-order derivative and the fractionalorder chaotic system model without equilibrium are introduced. In Section 3, dynamic analysis of the system is carried out at length. Section 4 gives the electronic circuit implementation results of the fractional-order system with hidden coexisting attractors. In Section 5 , based on the finitetime stable theorem, a finite-time synchronization of the proposed system is implemented. Section 6 summarizes the conclusions.

\section{Fractional-Order Form of the System without Equilibrium}

2.1. Definitions of Fractional-Order Derivative. So far, a few different definitions [29, 30] of fractional-order derivative have been proposed, such as Grunwald-Letnikov (G-L), Riemann-Liouville (R-L), Caputo, and Conformable definitions. In general, the first three definitions are extensively employed and the Caputo definition is more suitable for engineering applications. In this paper, we conduct the study on the basis of the Caputo definition, which is given by

$$
{ }_{a}^{C} D_{t}^{q} f(t)=\frac{1}{\Gamma(n-q)} \times \int_{a}^{t} \frac{f^{(n)}(\tau)}{(t-\tau)^{q+1-n}} \mathrm{~d} \tau
$$

In definition (1), $n$ is the first integer which is not less than $q$ and $n-1<q<n ; \Gamma(\cdot)$ is the Gamma function.

2.2. The Fractional-Order Chaotic System Model without Equilibrium. Sprott A system [31] is a three-dimensional conservative system and it has no equilibrium which depends on its third equation $\left(1-y^{2}\right)$. Based on Sprott A system, we construct a new 3D fractional-order chaotic system without equilibrium by imposing nonlinear functions associated with variables $x$ and $y$ on the third equation of Sprott A system, which can generate diverse coexisting attractors, described as

$$
\begin{aligned}
& \frac{\mathrm{d}^{q} x}{\mathrm{~d} t^{q}}=y \\
& \frac{\mathrm{d}^{q} y}{\mathrm{~d} t^{q}}=-x-y z \\
& \frac{\mathrm{d}^{q} z}{\mathrm{~d} t^{q}}=a x^{2}+b y^{2}+c x y+d
\end{aligned}
$$

where the parameters $a, b, c, d$ are real constants, $q$ is the fractional-order and $0<q<1$. When setting the system parameters and the fractional-order as $a=1.2, b=1, c=1$, $d=-1.3$ and $q=0.992$, the fractional-order system (2) with initial conditions $(x(0), y(0), z(0))=(1,1,0)$ presents hidden attractors as shown in Figure 1 and system (2) shows fourwing structure on the $x-z$ plane. The Poincaré map on the $x-z$ plane with $y=0$ shown in the three-dimensional space in Figure 2 also can verify it.

To further confirm the chaotic motion, by the BenettinWolf algorithm, the finite-time local Lyapunov exponents on the time interval $t \in[0,1500]$ with the same initial conditions and parameters as above are shown in Figure 3. And it can be seen that the largest one of the finite-time local LEs on the time interval $t \in[0,1500]$ always maintains to be positive.

Remark 1. In numerical simulations, the Lyapunov exponents may differ significantly from different trajectories and only a finite-time interval can be considered for their computation. Therefore, we follow the concept of the finite-time local Lyapunov exponents in the article.

\section{Dynamic Analysis of the Fractional-Order System without Equilibrium}

3.1. Basic Dynamic Properties. For system (2), it is symmetric about the $\mathrm{z}$-axis on account of the coordinate transformation $(x, y, z) \longrightarrow(-x,-y, z)$. And if let the right hand side of system (2) to zero, then it can be written as

$$
\begin{aligned}
y & =0 \\
-x-y z & =0 \\
a x^{2}+b y^{2}+c x y+d & =0
\end{aligned}
$$

While $x=y=0$, it is obvious that there is no equilibrium due to $d \neq 0$. Even so, through the above 


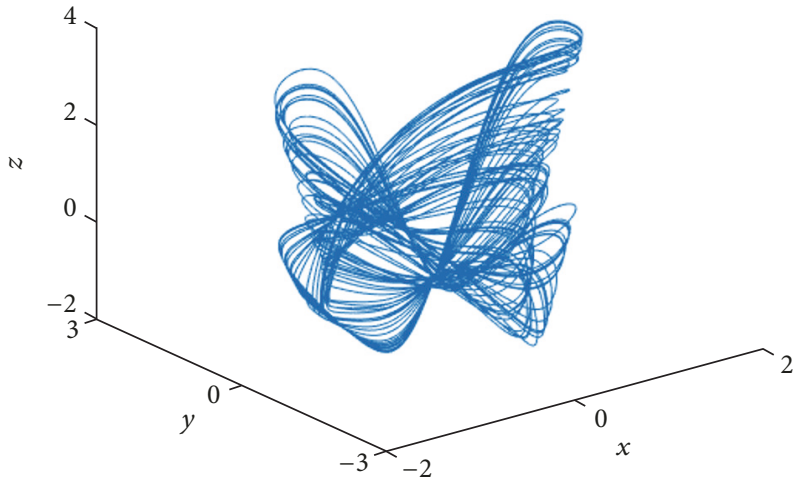

(a)

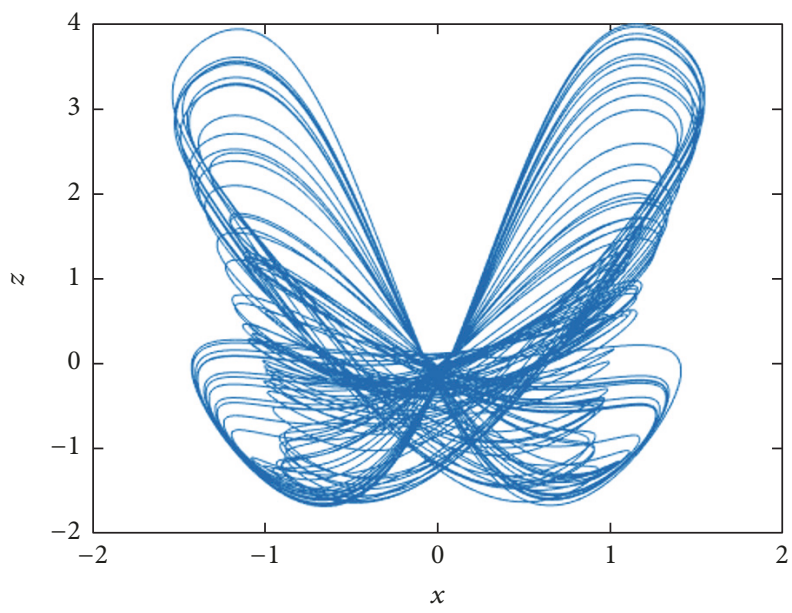

(c)

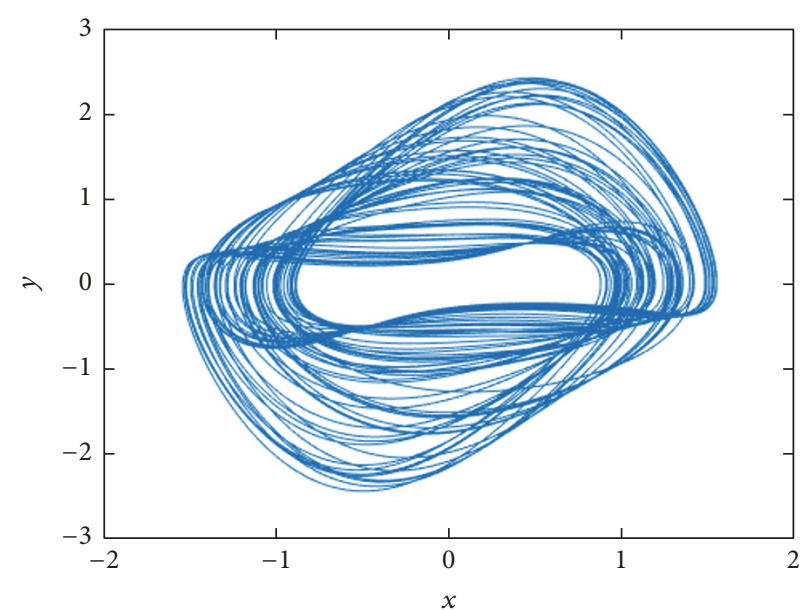

(b)

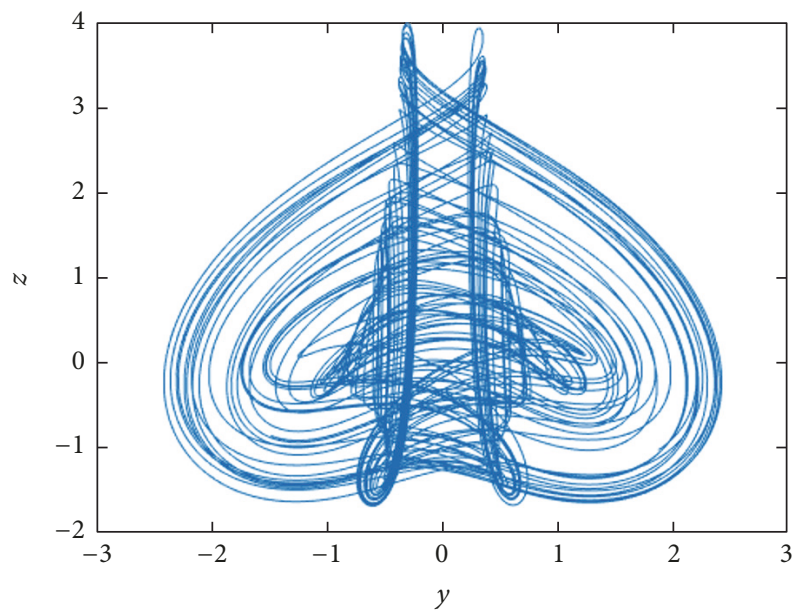

(d)

FIGURE 1: The chaotic attractor of the fractional-order system (2): (a) $x-y-z$ space; (b) $x-y$ plane; (c) $x-z$ plane; (d) $y-z$ plane.

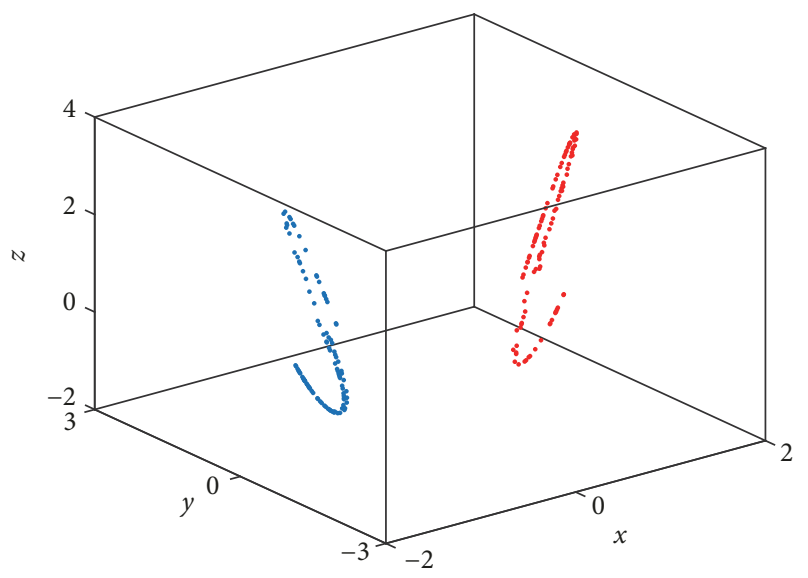

FIGURE 2: 3D Poincaré map of the fractional-order system (2) on the $x-z$ plane with $y=0$ (both directions through the cross-section in red and blue, respectively).

phase portraits, finite-time local Lyapunov exponents, and Poincaré map, the fractional-order system can show obvious chaotic characteristics and be regarded as the sort of hidden attractors.

3.2. Bifurcation Analysis of System Parameters. Bifurcation diagram can help us observe the further dynamic behaviors of the fractional-order system (2). While setting $b=1, c=1$, $d=-1.3$ and $q=0.99$, the bifurcation diagram and the largest finite-time local LEs varying with the parameter $a$ are shown in Figures 4 and 5. The performance via perioddoubling bifurcations and hidden coexisting attractors are observed in Figure 6. The fractional-order system presents several different symmetric pair of coexisting limit cycles for $a \leq 1.11$. As shown on Figures 6(a) and 6(b), there coexist a symmetric pair of limit cycles with period-1 orbits and another symmetric limit cycle with period-2 orbits, respectively. For $a>1.11$, the fractional system begins to exhibit obvious chaotic oscillations and hidden coexisting chaotic attractors while the parameter is not over 1.21 as shown in Figure 6(c).

When the parameters and fractional-order are chosen as $a=1.2, b=1, c=1$, and $q=0.99$, the bifurcation diagram of state variable $x$ varying with $d$ is provided in Figure 7 . 


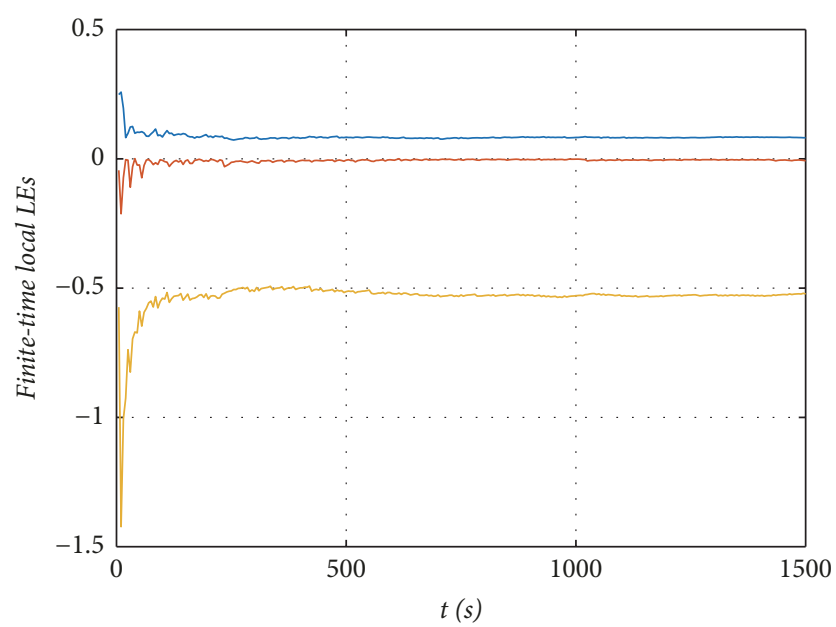

FIgURE 3: Dynamics of the finite-time local LEs of the fractionalorder system (2) with initial conditions $(x(0), y(0), z(0))=(1,1,0)$ on the time interval $t \in[0,1500]$.

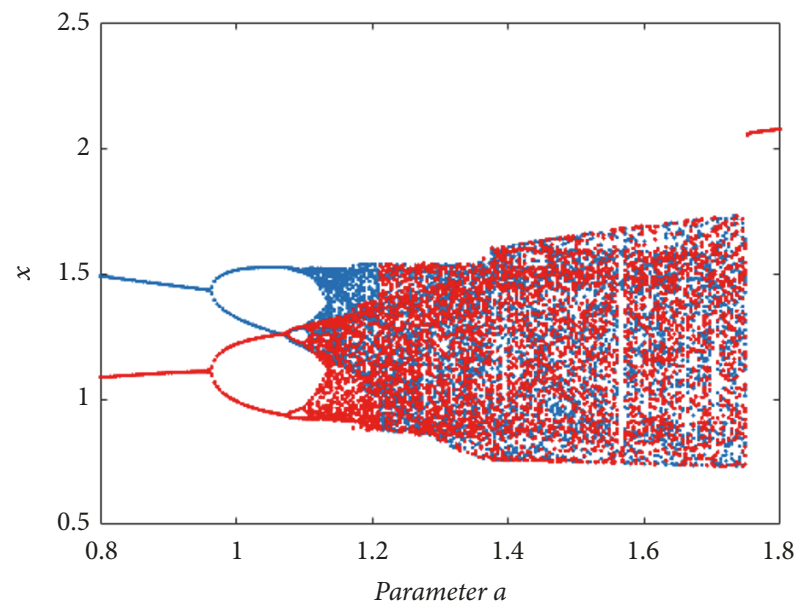

FIgURE 4: Bifurcation diagram with the variable parameter $a$, initial conditions $(x(0), y(0), z(0))=(1,1,0)$ (blue) and $(x(0), y(0), z(0))=$ $(-1,-1,0)$ (red).

Similarly, the states of the fractional-order system evolve from periodic state to multiperiodic state and then to chaos, which also exists periodic window in the neighborhood of $d=-1.2$. For purpose of further study in system parameter analysis, when keeping $b=1, c=1$ and $q=0.99$, Figure 8 plots the largest finite-time local LEs diagram with the mutual variation of parameters $a$ and $d$, and the right color bar denotes different levels of largest Lyapunov exponent. In Figure 8, different colors intuitively represent influences of parameters on dynamical behavior where the warmer colors mean the higher values of largest finite-time local LEs.

3.3. Influences of Fractional-Order $q$ and Initial Conditions. In Figure 9, keeping the system parameters as $a=1.2, b=1$, $c=1$, and $d=-1.3$, the bifurcation of system (2) with the change of fractional-order $q$ illuminates the system could undergo the period-doubling bifurcation and end up in chaos

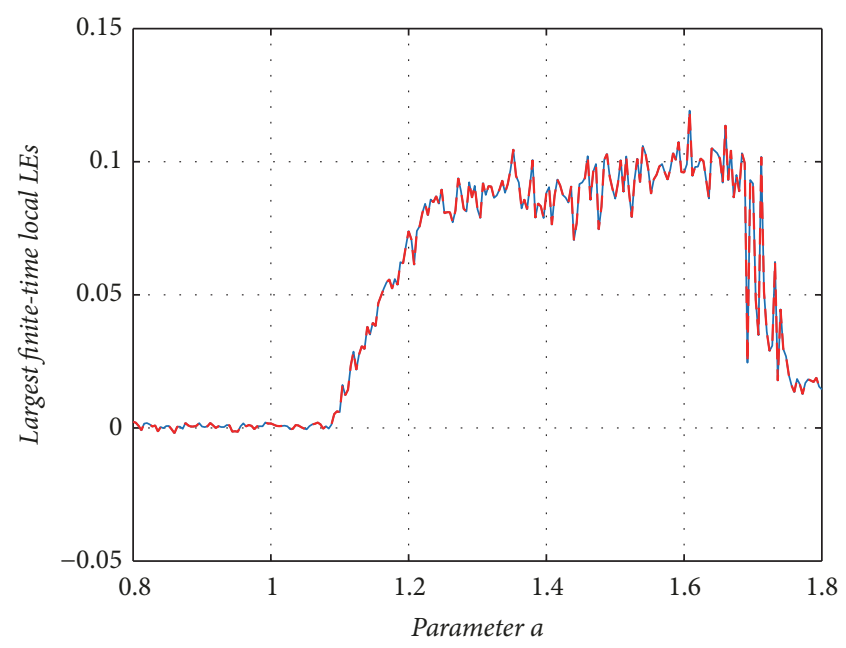

FIGURE 5: Largest finite-time local LEs on the time interval $t \in[0,1500]$ with the variable parameter $a$, initial conditions $(x(0), y(0), z(0))=$ $(1,1,0)$ (blue) and $(x(0), y(0), z(0))=(-1,-1,0)$ (red).

as the order is set on $q>0.982$. And there exists diverse coexisting attractors under different orders. For $q=0.99$, a symmetric pair of strange attractors coexist with different initial conditions as shown in Figures 6(c) and 11, and its basins of attractions in the $z=0$ plane are shown in Figure 10, where the one kind of chaotic attractor is distinguished from another by red and orange and the yellow region indicates initial conditions in it can reach unbounded orbits. For $q=$ 0.98 , there coexists a symmetric pair of limit cycles with period-4, as shown in Figure 12, and its basins in the $z=0$ plane are provided in Figure 13. Corresponding to the above analysis of basic dynamic properties, the basins have the similar symmetry about the $z$-axis.

Nevertheless, there are other cases of coexisting attractors. With order chosen as $q=0.999$, a strange attractor coexists with a limit cycle as shown in Figures 14 and 16, whose basins of attractions can be seen in Figure 15. The two attractors are respectively symmetric with respect to the $z$ axis and so do their basins. From the presented numerical results, the yellow region where initial values will lead to unbounded orbits largely remains invariant with different fractional orders, and the region of oscillation displays multitudinous and diverse dynamical behaviors.

\section{Circuit Implementation Results of the Fractional-Order System}

Circuit implementation of the fractional-order chaotic system plays a crucial role in actual application, which can also verify whether the results obtained by theory analysis and numerical computation are consistent and correct or not. Based on PSpice software, the schematic of the fractionalorder chaotic system with order $q=0.992$ and parameters $a=1.2, b=1, c=1$, and $d=-1.3$ is designed and implemented as shown in Figure 17. In Figure 17, AD633 and 


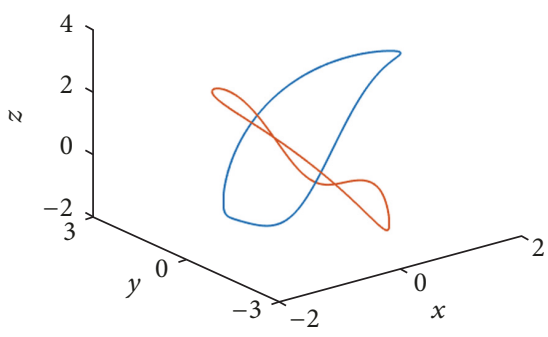

(a)

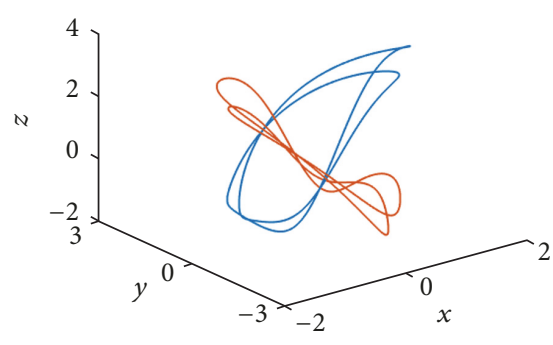

(b)

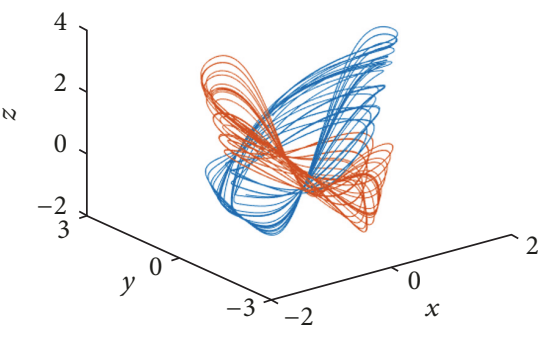

(c)

Figure 6: Coexisting attractors with initial conditions $(x(0), y(0), z(0))=(1,1,0)$ (blue) and $(x(0), y(0), z(0))=(-1,-1,0)$ (red) when the parameter $a$ is kept as (a) $a=0.9$; (b) $a=1.0$; (c) $a=1.2$.

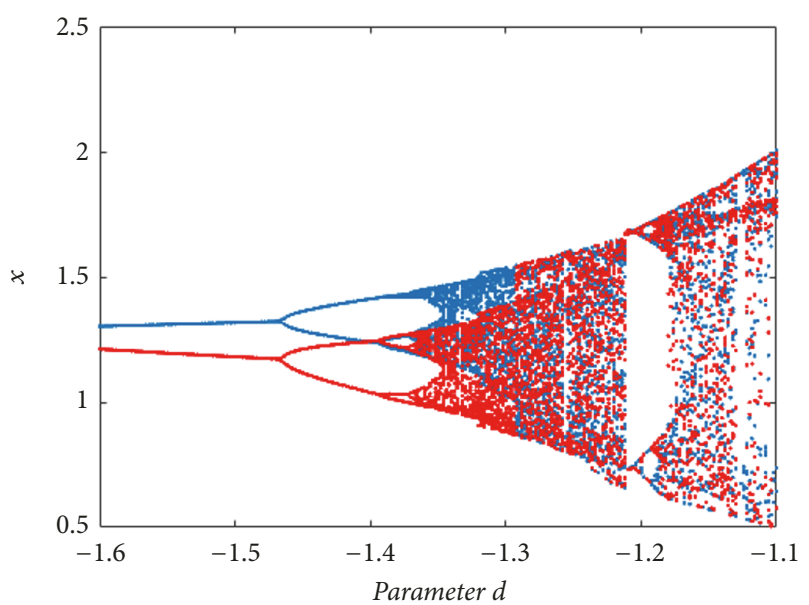

FIgURE 7: Bifurcation diagram with the variable parameter $d$, initial conditions $(x(0), y(0), z(0))=(1,1,0)$ (blue) and $(x(0), y(0), z(0))=$ $(-1,-1,0)(\mathrm{red})$.

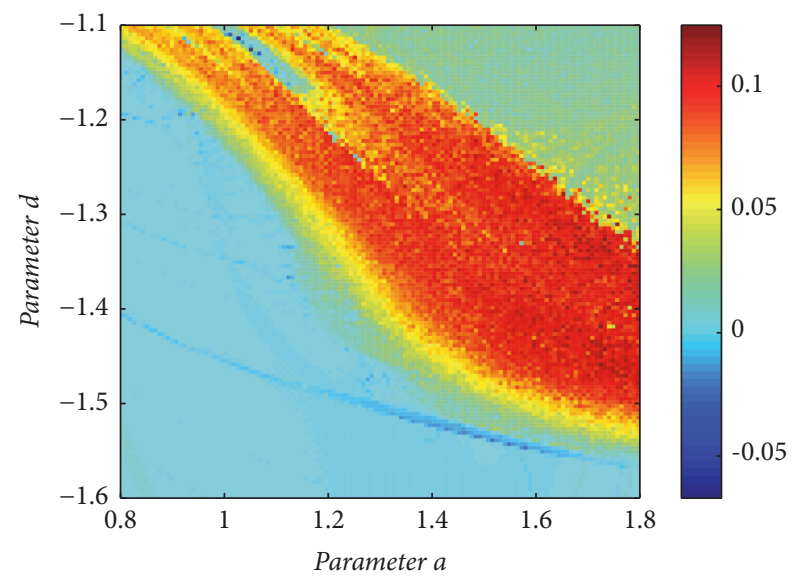

FIGURE 8: Dynamic map of Largest finite-time local LEs on the time interval $t \in[0,1500]$ with parameters $a$ and $b$ by selecting initial conditions $(x(0), y(0), z(0))=(1,1,0)$.

OP-07 are adopt as all analogue multipliers and operational amplifiers.

Therein, with the proposed transfer function approximations method [32] utilized and $\omega_{\max }=100, P_{T}=$ 0.01 assumed, the fractional integrator is derived by (4). In engineering practice it can be realized by three chain fractances composed of three resistor-capacitor pairs, as

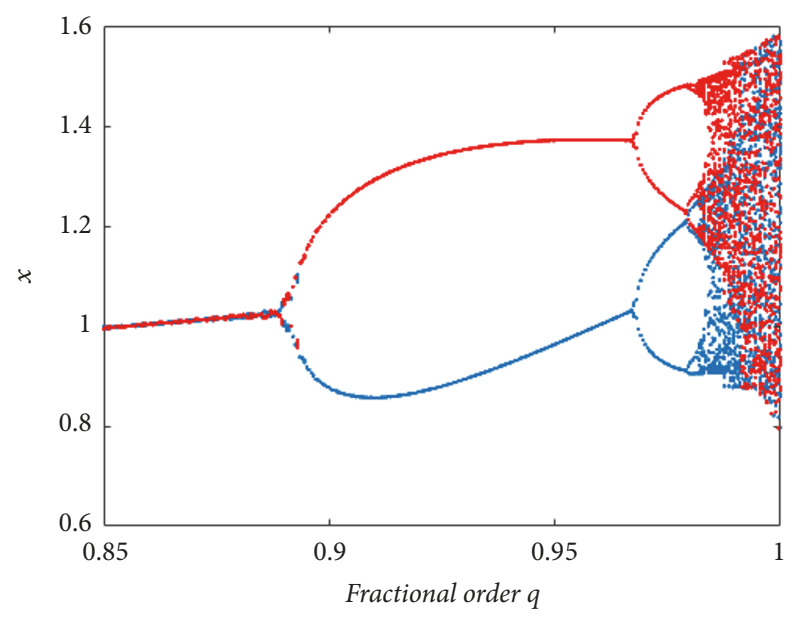

FIGURE 9: Bifurcation diagram with the variable fractional-order $q$, initial conditions $(x(0), y(0), z(0))=(1,1,0)$ (blue) and $(x(0), y(0), z(0))=(-1,-1,0)($ red $)$.

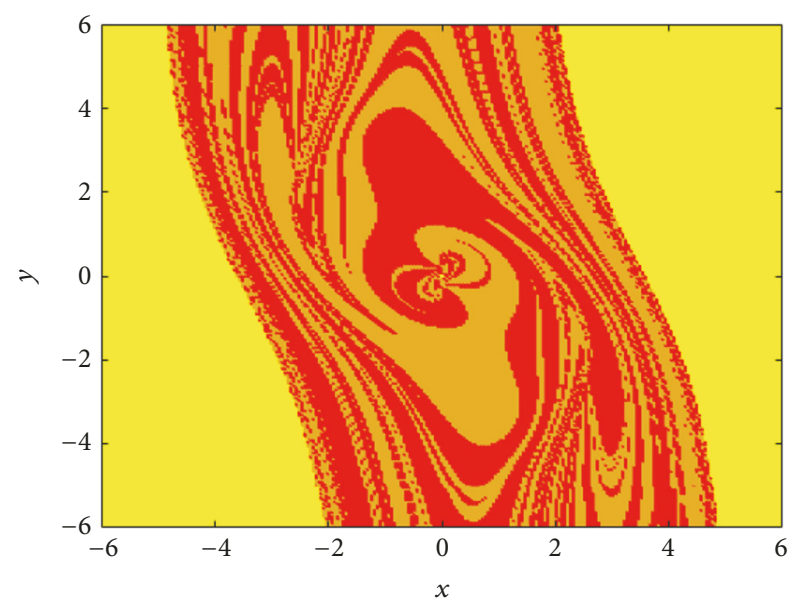

FIGURE 10: Cross-section for $z=0$ of the basins of attraction for the symmetric pair of strange attractors (red and orange) with fractional-order $q=0.99$ (the yellow region can reach unbounded orbits).

shown by (5). With comparing (4) with (5), the values of these circuit components are determined as $R_{a}=95957.169 \mathrm{k} \Omega$, $C_{a}=1.002 \mu \mathrm{F}, R_{b}=1.153 \mathrm{k} \Omega, C_{b}=13.893 \mu \mathrm{F}$ and $R_{c}=$ $0.205 \Omega, C_{c}=12.959 \mu \mathrm{F}$. 


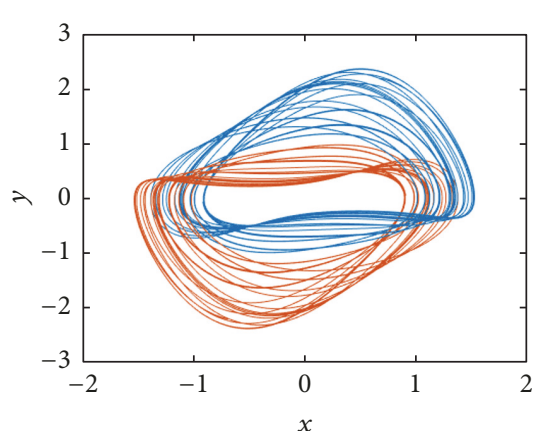

(a)

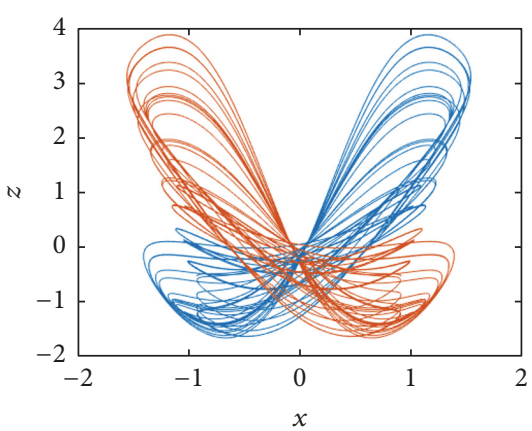

(b)

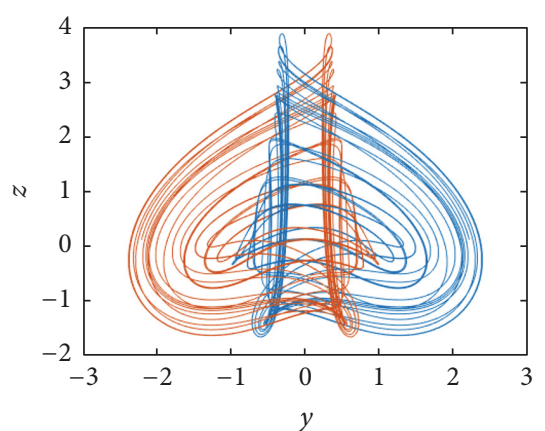

(c)

FIGURE 11: Coexisting chaotic attractors of the fractional-order system (2) with $q=0.99$, initial conditions $(x(0), y(0), z(0))=(1,1,0)($ blue $)$ and $(x(0), y(0), z(0))=(-1,-1,0)($ red): (a) $x-y$ plane; (b) $x-z$ plane; (c) $y$ - $z$ plane.

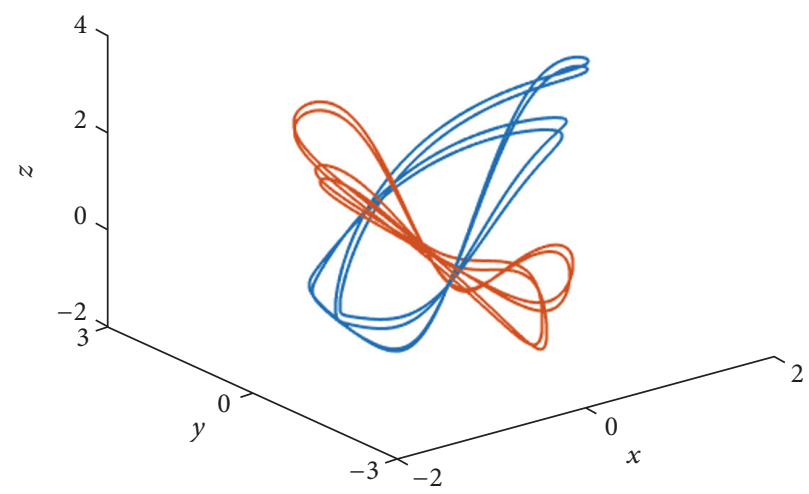

Figure 12: Coexisting limit cycles of the fractional-order system (2) with $q=0.98$, initial conditions $(x(0), y(0), z(0))=(1,1,0)($ blue $)$ and $(x(0), y(0), z(0))=(-1,-1,0)($ red $)$.

$$
\begin{aligned}
\frac{1}{s^{0.99}} & =\frac{1.1471(s+58.2265)\left(s+3.5105 \times 10^{5}\right)}{(s+0.0104)(s+62.4256)\left(s+3.7636 \times 10^{5}\right)} \\
F(s) & =\frac{1 / C_{a}}{s+1 / R_{a} C_{a}}+\frac{1 / C_{b}}{s+1 / R_{b} C_{b}}+\frac{1 / C_{c}}{s+1 / R_{c} C_{c}}=\frac{1}{C_{0}} \\
& \frac{\left(C_{0} / C_{a}+C_{0} / C_{b}+C_{0} / C_{c}\right)\left[s^{2}+\left(\left(\left(C_{b}+C_{c}\right) / R_{a}+\left(C_{a}+C_{c}\right) / R_{b}+\left(C_{a}+C_{b}\right) / R_{c}\right) /\left(C_{a} C_{b}+C_{b} C_{c}+C_{a} C_{c}\right)\right) s+\left(\left(R_{a}+R_{b}+R_{c}\right) / R_{a} R_{b} R_{c}\right) /\left(C_{a} C_{b}+C_{b} C_{c}+C_{a} C_{c}\right)\right]}{\left(s+1 / R_{a} C_{a}\right)\left(s+1 / R_{b} C_{b}\right)\left(s+1 / R_{c} C_{c}\right)}
\end{aligned}
$$

With the Kirchhoff's circuit laws, the mathematical model of system (2) is described by the circuit equations as follow:

$$
\begin{aligned}
\frac{\mathrm{d}^{q} x}{\mathrm{~d} t^{q}}= & \frac{R_{7}}{R_{1} R_{6} C_{0}} y \\
\frac{\mathrm{d}^{q} y}{\mathrm{~d} t^{q}}= & -\frac{1}{R_{3} C_{0}} x-\frac{1}{\eta R_{2} C_{0}} y z \\
\frac{\mathrm{d}^{q} z}{\mathrm{~d} t^{q}}= & \frac{R_{5}}{\eta R_{8} R_{4} C_{0}} x^{2}+\frac{R_{7}}{\eta R_{9} R_{6} C_{0}} y^{2}+\frac{R_{7}}{\eta R_{10} R_{6} C_{0}} x y \\
& +\frac{R_{12}}{R_{13} R_{11} C_{0}} V_{\mathrm{CC}-}
\end{aligned}
$$

where $C_{0}=1 \mu \mathrm{F}$ and $1 / \eta=1 / 10$ hinged on the characteristic of $\mathrm{AD} 633$. The parameters of electronic components are calculated as: $V_{\mathrm{CC}+}=-V_{\mathrm{CC}-}=15 \mathrm{~V}_{\mathrm{DC}}, R_{1}=R_{3}=$ $R_{11}=1000 \mathrm{k} \Omega, R_{2}=R_{4}=R_{5}=R_{6}=R_{7}=R_{9}=$ $R_{10}=100 \mathrm{k} \Omega, R_{8}=83.33 \mathrm{k} \Omega, R_{12}=10 \mathrm{k} \Omega, R_{13}=$ $115.38 \mathrm{k} \Omega$. Figure 18 demonstrates circuit implementation results in which hidden strange attractors can been observed distinctly. And it also indicates that the circuital results are comparatively consistent with the numerical simulations.

\section{Finite-Time Synchronization of the Fractional-Order System with Hidden Coexisting Attractors}

There is a wide variety of literature [24, 33-37] on synchronization of fractional-order chaotic systems by reason of comprehensive practical application in many fields, such as aerospace, power system, secure communication and so 


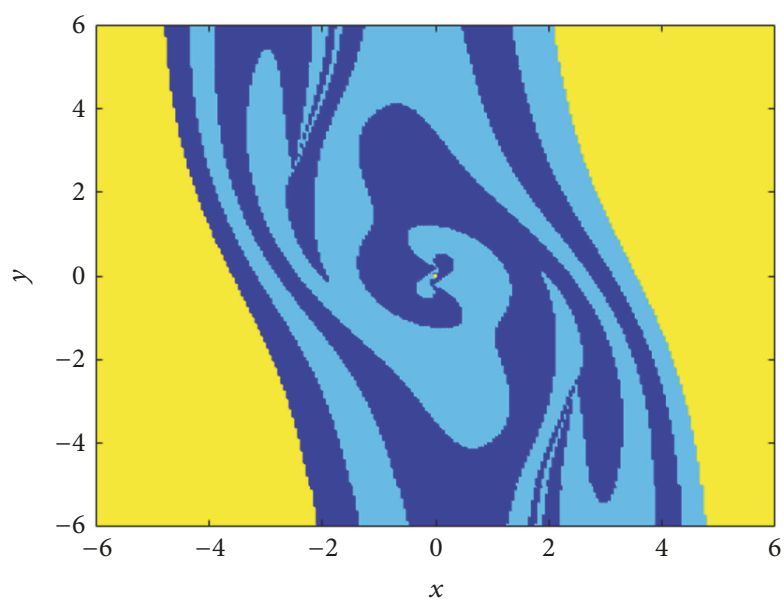

Figure 13: Cross-section for $z=0$ of the basins of attraction for the symmetric pair of limit cycles (blue and cyan) with fractional-order $q=0.98$ (the yellow region can reach unbounded orbits).

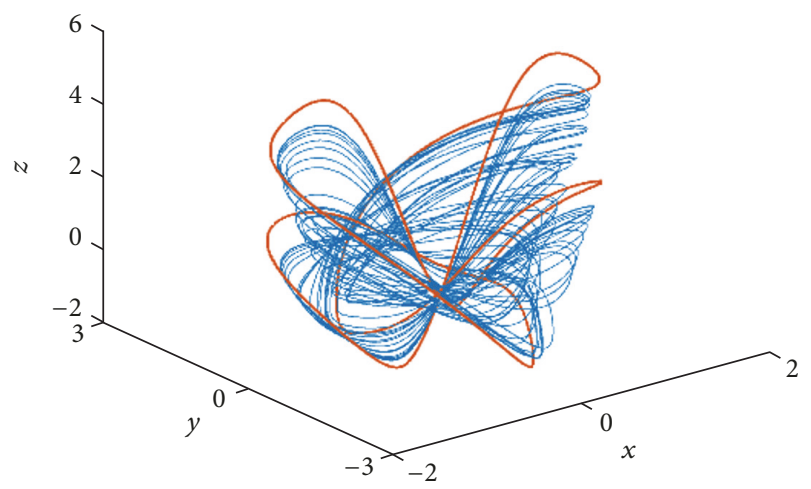

FIgURE 14: Coexistence of a chaotic attractor and a limit cycle of system (2) with $q=0.999$, initial conditions $(x(0), y(0), z(0))=$ $(1,1,0)$ (blue) and $(x(0), y(0), z(0))=(-0.5,5,0)$ (red).

on. Research about finite-time synchronization in allusion to fractional-order chaotic system with hidden attractors, are quite limited. In this section, based on the finite-time stable theorem [38], in consideration of the disturbances, the modified controller is devised for synchronization of the fractional-order system with hidden chaotic attractors and achieves the anticipated effect.

Theorem 2 (see [38]). For the fractional-order system in general, if it satisfies

$$
\begin{array}{r}
\boldsymbol{x}_{a}^{\mathrm{C}} D_{t}^{q} \boldsymbol{x}^{\mathrm{T}}=\frac{\Gamma(2)}{\Gamma(2+q)}{ }_{a}^{C} D_{t}^{q} \boldsymbol{x}\left(\boldsymbol{x}^{q}\right)^{\mathrm{T}} \leq-k\left(\boldsymbol{x} \boldsymbol{x}^{\mathrm{T}}\right)^{\alpha}, \\
\quad \alpha<\frac{q+q^{2}}{2}, k>0,
\end{array}
$$

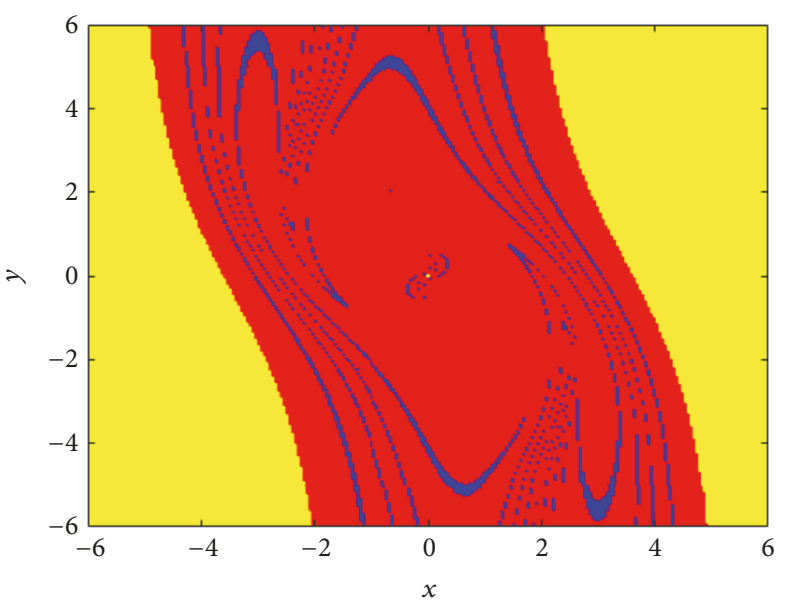

Figure 15: Cross-section for $z=0$ of the basins of attraction for the chaotic attractor (red) and the limit cycle (blue) with fractionalorder $q=0.999$ (the yellow region can reach unbounded orbits).

where $\boldsymbol{x}=\left[x_{1}, x_{2}, \cdots x_{n}\right], \boldsymbol{x}^{q}=\left[x_{1}^{q}, x_{2}^{q}, \cdots x_{n}^{q}\right]$. Then the state variables $\boldsymbol{x}$ of the given fractional-order system will converge to zero within a finite-time:

$$
\begin{aligned}
t= & {\left[v(0)^{q-2 \alpha /(1+q)}\right.} \\
& \left.\cdot \frac{\Gamma(1+q-2 \alpha /(1+q)) \Gamma(1+q)}{\Gamma(1+2 q-2 \alpha /(1+q)) k \Gamma(2+q)}\right]^{1 / q},
\end{aligned}
$$

where $v=\boldsymbol{x}\left(\boldsymbol{x}^{q}\right)^{\mathrm{T}}$.

Lemma 3. $\forall a, b>0,0<c<1$ :

$$
(a+b)^{c} \leq a^{c}+b^{c}
$$

Making allowances for the internal disturbances $\Delta f_{i}^{m}(\boldsymbol{X}, t)$ and $\Delta f_{i}^{s}\left(\boldsymbol{X}_{1}, t\right)\left(i=1,2,3\right.$ and $\left.x, y, z \in \boldsymbol{X}, x_{1}, y_{1}, z_{1} \in \boldsymbol{X}_{1}\right)$ in the fractional-order chaotic system (2), the master system and the slave system can be obtained as

$$
\begin{aligned}
& \frac{\mathrm{d}^{q} x}{\mathrm{~d} t^{q}}=y+\Delta f_{1}^{m}(\boldsymbol{X}, t) \\
& \frac{\mathrm{d}^{q} y}{\mathrm{~d} t^{q}}=-x-y z+\Delta f_{2}^{m}(\boldsymbol{X}, t) \\
& \frac{\mathrm{d}^{q} z}{\mathrm{~d} t^{q}}=a x^{2}+b y^{2}+c x y+d+\Delta f_{3}^{m}(\boldsymbol{X}, t) \\
& \frac{\mathrm{d}^{q} x_{1}}{\mathrm{~d} t^{q}}=y_{1}+\Delta f_{1}^{s}\left(\boldsymbol{X}_{1}, t\right)-u_{1} \\
& \frac{\mathrm{d}^{q} y_{1}}{\mathrm{~d} t^{q}}=-x_{1}-y_{1} z_{1}+\Delta f_{2}^{s}\left(\boldsymbol{X}_{1}, t\right)-u_{2} \\
& \frac{\mathrm{d}^{q} z_{1}}{\mathrm{~d} t^{q}}=a x_{1}^{2}+b y_{1}^{2}+c x_{1} y_{1}+d+\Delta f_{3}^{s}\left(\boldsymbol{X}_{1}, t\right)-u_{3}
\end{aligned}
$$

where $a=1.2, b=1, c=1, d=-1.3$, and $q=0.99 ; \boldsymbol{u}=$ $\left[u_{1}, u_{2}, u_{3}\right]$ is the designed controller. 


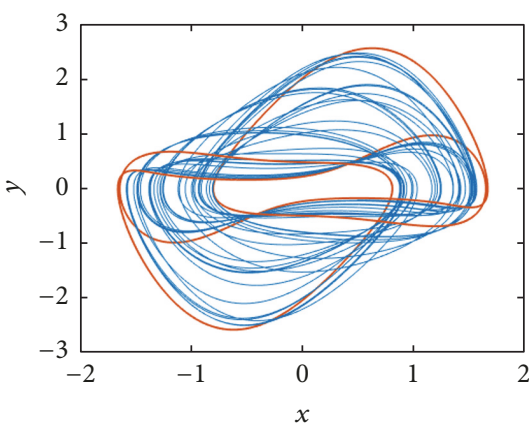

(a)

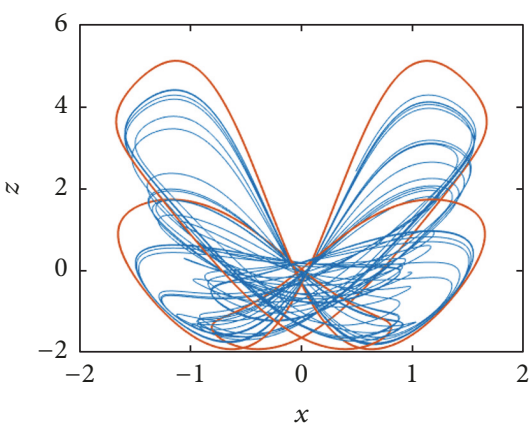

(b)

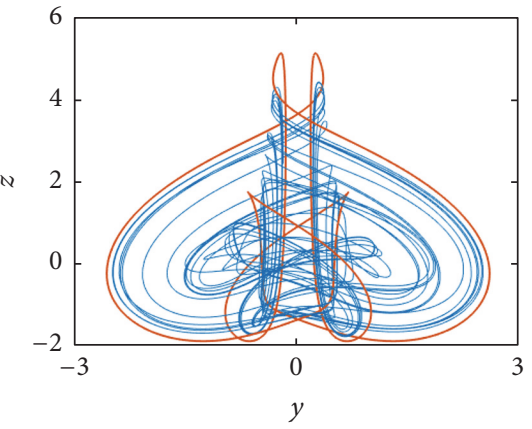

(c)

FIGURE 16: Coexistence of a chaotic attractor and a limit cycle of system (2) with $q=0.999$, initial conditions $(x(0), y(0), z(0))=(1,1,0)$ (blue) and $(x(0), y(0), z(0))=(-0.5,5,0)$ (red): (a) $x-y$ plane; (b) $x-z$ plane; (c) $y$ - $z$ plane.

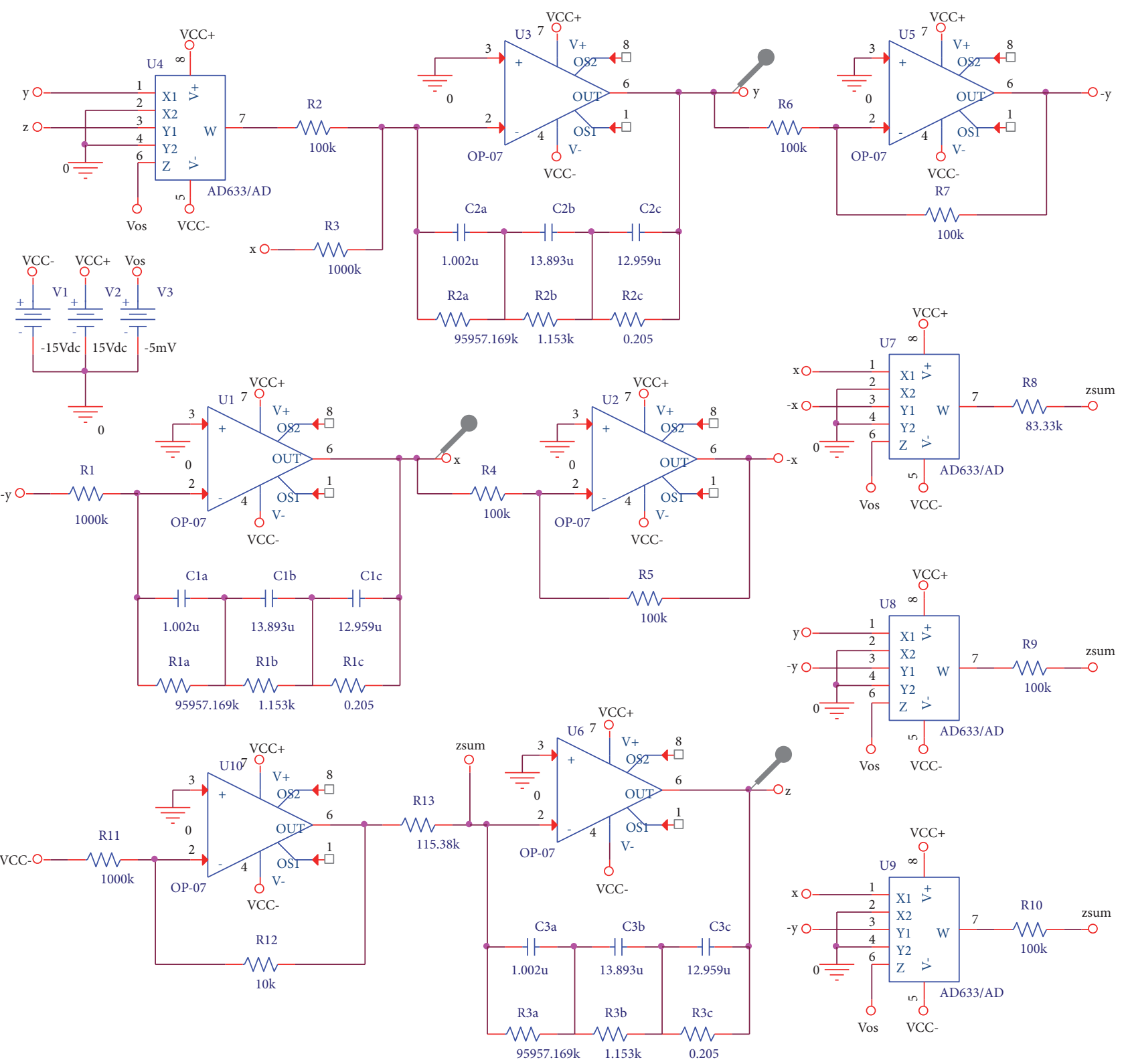

FIGURE 17: The electronic circuit schematic of the fractional-order system (2) in PSpice. 


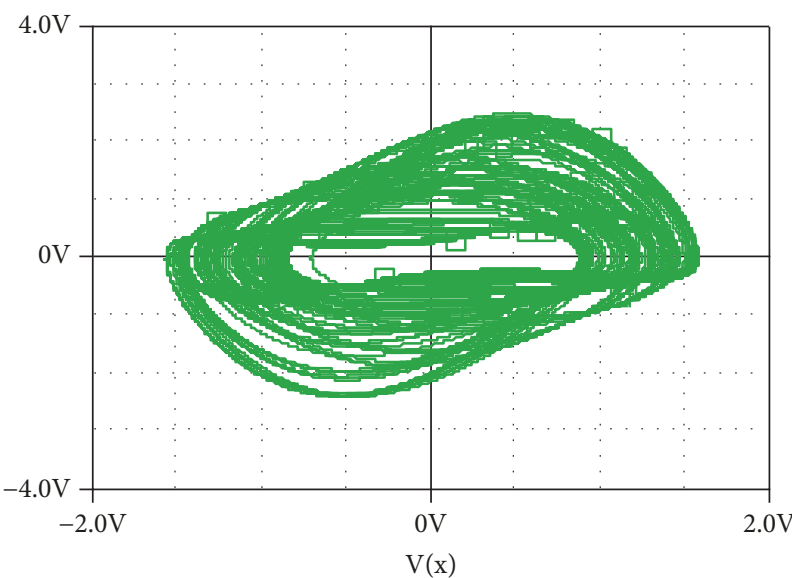

$\mathrm{V}(\mathrm{y})$

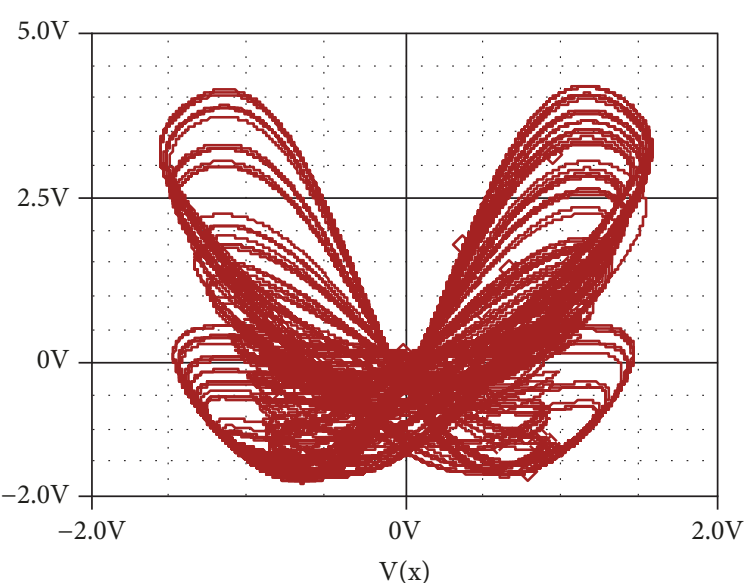

$\diamond \mathrm{V}(\mathrm{z})$

(a)

(b)

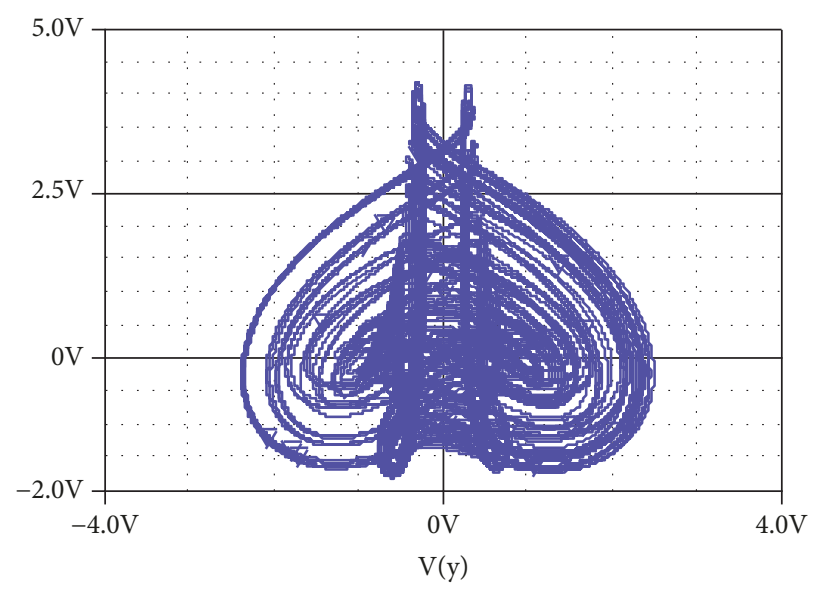

$\nabla \mathrm{V}(\mathrm{z})$

(c)

FIGURE 18: Hidden chaotic attractors of the fractional-order system (2) in PSpice: (a) on the $x-y$ plane; (b) on the $x-z$ plane; (c) on the $y-z$ plane.

Assumption 4. The disturbances $\Delta f_{i}^{m}(\boldsymbol{X}, t), \Delta f_{i}^{s}\left(\boldsymbol{X}_{1}, t\right)$ are bounded. In other words, positive constants $F_{i}^{m}$ and $F_{i}^{s}$ can be found to satisfy

$$
\begin{aligned}
& \left|\Delta f_{i}^{m}(\boldsymbol{X}, t)\right| \leq F_{i}^{m}, \\
& \left|\Delta f_{i}^{s}\left(\boldsymbol{X}_{1}, t\right)\right| \leq F_{i}^{s}
\end{aligned}
$$

Afterwards, we define the error system as $e_{1}=x_{1}-x$, $e_{2}=y_{1}-y, e_{3}=z_{1}-z$, which can ultimately be derived as

$$
\begin{aligned}
\frac{\mathrm{d}^{q} e_{1}}{\mathrm{~d} t^{q}}= & e_{2}+\Delta f_{1}^{s}\left(\boldsymbol{X}_{1}, t\right)-\Delta f_{1}^{m}(\boldsymbol{X}, t)-u_{1} \\
\frac{\mathrm{d}^{q} e_{2}}{\mathrm{~d} t^{q}}= & -e_{1}-y_{1} e_{3}-z e_{2}+\Delta f_{2}^{s}\left(\boldsymbol{X}_{1}, t\right)-\Delta f_{2}^{m}(\boldsymbol{X}, t) \\
& -u_{2} \\
\frac{\mathrm{d}^{q} e_{3}}{\mathrm{~d} t^{q}}= & 1.2 x_{1} e_{1}+1.2 x e_{1}+y_{1} e_{2}+y e_{2}+x_{1} e_{2}+y e_{1} \\
& +\Delta f_{3}^{s}\left(\boldsymbol{X}_{1}, t\right)-\Delta f_{3}^{m}(\boldsymbol{X}, t)-u_{3}
\end{aligned}
$$

Theorem 5. It can realize the synchronization of the master system (10) and the slave system (11) within a finite-time, while the control input is provided as

$$
\begin{aligned}
& u_{1}=1.2 x_{1} e_{3}+y e_{3}+k e_{1}^{\beta}+k_{1} \operatorname{sign}\left(e_{1}\right) \\
& u_{2}=-z e_{2}+x_{1} e_{3}+k e_{2}^{\beta}+k_{2} \operatorname{sign}\left(e_{2}\right) \\
& u_{3}=1.2 x e_{1}+y e_{2}+k e_{3}^{\beta}+k_{3} \operatorname{sign}\left(e_{3}\right)
\end{aligned}
$$

where $k_{i}=F_{i}^{s}+F_{i}^{m}, i=1,2,3$.

Proof. According to (7) and (12), the corresponding function with the error system (13) and the control input (14) can be constructed as

$$
\begin{aligned}
& \frac{\Gamma(2)}{\Gamma(2+q)} \frac{\mathrm{d}^{q}}{\mathrm{~d} t^{q}}\left[\begin{array}{lll}
e_{1} & e_{2} & e_{3}
\end{array}\right]\left[\begin{array}{lll}
e_{1}^{q} & e_{2}^{q} & e_{3}^{q}
\end{array}\right]^{\mathrm{T}} \\
& =\left[\begin{array}{lll}
e_{1} & e_{2} & e_{3}
\end{array}\right]\left[\begin{array}{lll}
\frac{\mathrm{d}^{q}}{\mathrm{~d} t^{q}} e_{1} & \frac{\mathrm{d}^{q}}{\mathrm{~d} t^{q}} e_{2} & \frac{\mathrm{d}^{q}}{\mathrm{~d} t^{q}} e_{3}
\end{array}\right]^{\mathrm{T}}
\end{aligned}
$$




$$
\begin{aligned}
= & e_{1} e_{2}-e_{1} e_{2}-y_{1} e_{2} e_{3}-z e_{2}^{2}+1.2 x_{1} e_{1} e_{3}+1.2 x e_{1} e_{3} \\
& +y_{1} e_{2} e_{3}+y e_{2} e_{3}+x_{1} e_{2} e_{3}+y e_{1} e_{3} \\
& +\left(\Delta f_{1}^{s}\left(\boldsymbol{X}_{1}, t\right)-\Delta f_{1}^{m}(\boldsymbol{X}, t)\right) e_{1} \\
& +\left(\Delta f_{2}^{s}\left(\boldsymbol{X}_{1}, t\right)-\Delta f_{2}^{m}(\boldsymbol{X}, t)\right) e_{2} \\
& +\left(\Delta f_{3}^{s}\left(\boldsymbol{X}_{1}, t\right)-\Delta f_{3}^{m}(\boldsymbol{X}, t)\right) e_{3}-u_{1} e_{1}-u_{2} e_{2} \\
& -u_{3} e_{3} \\
= & -k e_{1}^{\beta+1}-k e_{2}^{\beta+1}-k e_{3}^{\beta+1} \\
& +\sum_{i=1}^{3}\left(\left(\Delta f_{i}^{s}\left(\boldsymbol{X}_{1}, t\right)-\Delta f_{i}^{m}(\boldsymbol{X}, t)\right) e_{i}-k_{i} e_{i} \operatorname{sign}\left(e_{i}\right)\right) \\
\leq & -k e_{1}^{\beta+1}-k e_{2}^{\beta+1}-k e_{3}^{\beta+1} \\
& +\sum_{i=1}^{3}\left(\left(F_{i}^{s}+F_{i}^{m}\right) e_{i}-k_{i} e_{i} \operatorname{sign}\left(e_{i}\right)\right) \\
= & -k e_{1}^{\beta+1}-k e_{2}^{\beta+1}-k e_{3}^{\beta+1}+\sum_{i=1}^{3}\left(k_{i}\left(e_{i}-e_{i} \operatorname{sign}\left(e_{i}\right)\right)\right) \\
\leq & -k e_{1}^{\beta+1}-k e_{2}^{\beta+1}-k e_{3}^{\beta+1}
\end{aligned}
$$

where $e_{i}-e_{i} \operatorname{sign}\left(e_{i}\right)=e_{i}-\left|e_{i}\right| \leq 0$.

By reference to Lemma 3, (15) can be derived as

$$
\begin{aligned}
- & k e_{1}^{\beta+1}-k e_{2}^{\beta+1}-k e_{3}^{\beta+1} \\
& =-k\left(e_{1}^{2}\right)^{(\beta+1) / 2}-k\left(e_{2}^{2}\right)^{(\beta+1) / 2}-k\left(e_{3}^{2}\right)^{(\beta+1) / 2} \\
& \leq-k\left(e_{1}^{2}+e_{2}^{2}+e_{3}^{2}\right)^{(\beta+1) / 2}=-k\left(\boldsymbol{e} e^{\mathrm{T}}\right)^{(\beta+1) / 2}
\end{aligned}
$$

Therefore, from Theorem 2, the error system (13) states will converge to zero within a finite-time.

The numerical simulations are carried out to verify the effectiveness of the proposed scheme. The disturbances $\Delta f_{i}^{m}(\boldsymbol{X}, t), \Delta f_{i}^{s}\left(\boldsymbol{X}_{1}, t\right)$ are chosen as (17). The parameters for controllers are set as $k=1.5, \beta=0.5$ and $k_{1}=1.0$, $k_{2}=1.4, k_{3}=4.4$. Initial states of the master system and the slave system are selected as $(x(0), y(0), z(0))=$ $(-0.5,-0.5,2)$ and $\left(x_{1}(0), y_{1}(0), z_{1}(0)\right)=(1,1,0)$. As shown in Figure 19, the error system (13) can stabilize to zero within 1s. Meanwhile, time response of the master system (10) and the slave system (11) under control input (14) is presented in Figure 20 and the system states of the slave system will track the trajectories of the master system within 1s. In comparison with the original controllers which do not take into account disturbance factors in [38], the improved controllers in this paper can synchronize the master system and the slave system under disturbances. The simulation results in Figure 20 also indicate that the proposed method is fast and can achieve chaos synchronization within finitetime. All the results verify the effectiveness of the proposed

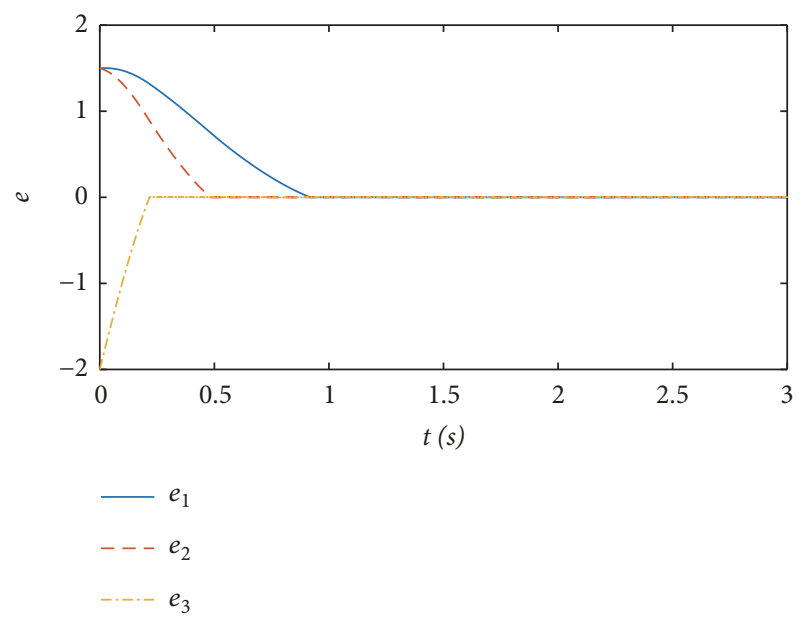

FIGURE 19: Synchronization errors between the master and slave systems.

scheme in synchronization of fractional-order chaotic system with hidden coexisting attractors.

$$
\begin{aligned}
& \Delta f_{1}^{m}(\boldsymbol{X}, t)=0.15 x \cos (2 t), \\
& \Delta f_{1}^{s}\left(\boldsymbol{X}_{1}, t\right)=-0.2 x_{1} \sin (2 t) \\
& \Delta f_{2}^{m}(\boldsymbol{X}, t)=-0.2 y \sin (3 t), \\
& \Delta f_{2}^{s}\left(\boldsymbol{X}_{1}, t\right)=0.1 y_{1} \sin (2 t) \\
& \Delta f_{3}^{m}(\boldsymbol{X}, t)=0.1 z \cos (t) \\
& \Delta f_{3}^{s}\left(\boldsymbol{X}_{1}, t\right)=0.15 z_{1} \cos (t)
\end{aligned}
$$

\section{Conclusions}

In this paper, a novel three-dimensional symmetric fractionalorder system without equilibrium, which can generate various hidden coexisting attractors, has been introduced. We have analyzed dynamical characteristics of the fractionalorder system in detail by numerical simulations and found the system presents abundant complex dynamic characteristics. Especially, through bifurcation diagram, the basins of attraction, and phase diagram, the influence of the order on dynamic behavior of the fractional-order system has been explored in depth. With the approximations of fractionalorder integrator $q=0.992$ derived, circuit implementation of the fractional-order system with hidden coexisting attractors has been achieved in PSpice and it has a good agreement with the numerical simulations. Furthermore, the modified controller has been devised and proved, which can realize the finite-time synchronization of the fractional-order system without equilibrium under the disturbances. Finally, simulation results also illustrate the robustness and speediness of the proposed controller. In order to simplify control inputs, fractional-order finite-time backstepping method and dynamic surface method will be considered in the future. Furthermore, secure communication and image encryption 


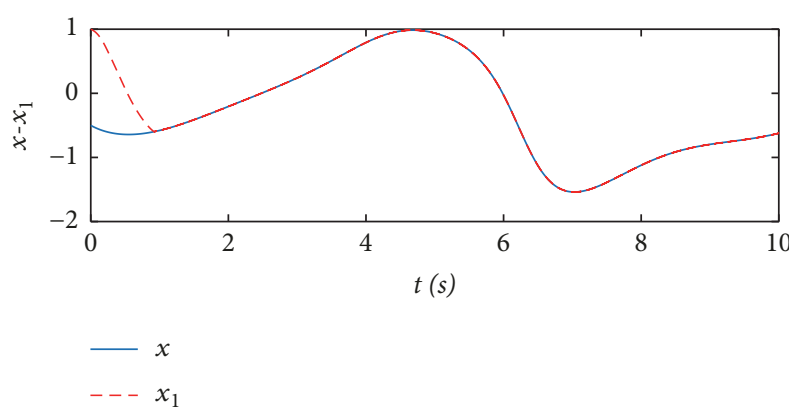

(a)

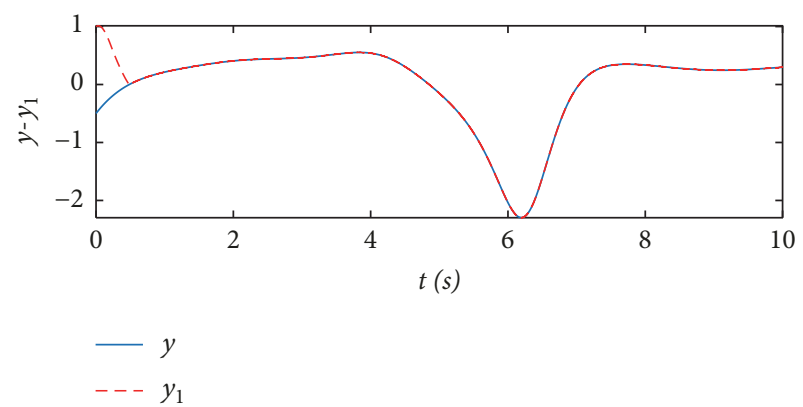

(b)

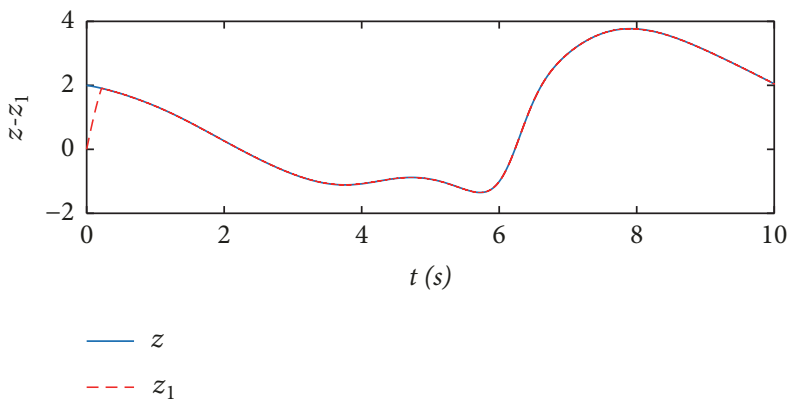

(c)

FIGURE 20: Synchronization results of the master and slave systems: (a) $x-x_{1}$; (b) $y-y_{1}$; (c) $z-z_{1}$.

based on the novel fractional-order chaotic system with hidden coexisting attractors will also be further investigated.

\section{Data Availability}

The data used to support the findings of this study are included within the article.

\section{Conflicts of Interest}

There are no conflicts of interest regarding the publication of this paper.

\section{Acknowledgments}

This project was supported by the National Natural Science Foundation of China (Grant no. 51877162).

\section{References}

[1] E. N. Lorenz, "Deterministic nonperiodic flow," Journal of the Atmospheric Sciences, vol. 20, no. 2, pp. 130-141, 1963.

[2] O. E. Rössler, "An equation for continuous chaos," Physics Letters A, vol. 57, no. 5, pp. 397-398, 1976.

[3] G. Chen and T. Ueta, "Yet another chaotic attractor," International Journal of Bifurcation and Chaos, vol. 9, no. 7, pp. 14651466, 1999.

[4] J. Lü and G. Chen, "A new chaotic attractor coined," International Journal of Bifurcation and Chaos, vol. 12, no. 3, pp. 659661, 2002.

[5] C. Liu, T. Liu, L. Liu, and K. Liu, "A new chaotic attractor," Chaos, Solitons \& Fractals, vol. 22, no. 5, pp. 1031-1038, 2004.
[6] G. A. Leonov, N. V. Kuznetsov, and V. I. Vagaitsev, "Localization of hidden Chua's attractors," Physics Letters A, vol. 375, no. 23, pp. 2230-2233, 2011.

[7] G. A. Leonov, N. V. Kuznetsov, and T. N. Mokaev, "Hidden attractor and homoclinic orbit in Lorenz-like system describing convective fluid motion in rotating cavity," Communications in Nonlinear Science and Numerical Simulation, vol. 28, no. 1-3, pp. 166-174, 2015.

[8] X. Wang and G. R. Chen, "A chaotic system with only one stable equilibrium," Communications in Nonlinear Science and Numerical Simulation, vol. 17, no. 3, pp. 1264-1272, 2012.

[9] M. Molaie, S. Jafari, J. C. Sprott, and S. M. R. H. Golpayegani, "Simple chaotic flows with one stable equilibrium," International Journal of Bifurcation and Chaos, vol. 23, no. 11, Article ID 1350188, 2013.

[10] S. Jafari and J. C. Sprott, "Simple chaotic flows with a line equilibrium," Chaos, Solitons \& Fractals, vol. 57, no. 4, pp. 79-84, 2013.

[11] S. Jafari, A. Ahmadi, A. J. M. Khalaf, H. R. Abdolmohammadi, V.-T. Pham, and F. E. Alsaadi, "A new hidden chaotic attractor with extreme multi-stability," AEÜ - International Journal of Electronics and Communications, vol. 89, pp. 131-135, 2018.

[12] Z. C. Wei, "Dynamical behaviors of a chaotic system with no equilibria," Physics Letters A, vol. 376, no. 2, pp. 102-108, 2011.

[13] S. Jafari, J. C. Sprott, and S. M. R. H. Golpayegani, "Elementary quadratic chaotic flows with no equilibria," Physics Letters A, vol. 377, no. 9, pp. 699-702, 2013.

[14] C. B. Li, J. C. Sprott, and H. Xing, "Hypogenetic chaotic jerk flows," Physics Letters A, vol. 380, no. 11-12, pp. 1172-1177, 2016.

[15] X. Y. Hu, C. X. Liu, L. Liu, Y. P. Yao, and G. C. Zheng, "Multiscroll hidden attractors and multi-wing hidden attractors in a 
5-dimensional memristive system," Chinese Physics B, vol. 26, no. 11, Article ID 110502, 2017.

[16] M. F. Danca, "Hidden chaotic attractors in fractional-order systems," Nonlinear Dynamics, vol. 89, no. 1, pp. 577-586, 2017.

[17] C. B. Li and J. C. Sprott, "Coexisting hidden attractors in a 4-D simplified Lorenz system," International Journal of Bifurcation \& Chaos, vol. 24, no. 3, Article ID 1450034, 2014.

[18] J. Ruan Y, K. Sun H, and J. Mou, "Memristor-based Lorenz hyper-chaotic system and its circuit implementation," Acta Physica Sinica, vol. 65, no. 19, Article ID 190502, 2016.

[19] B. C. Bao, H. Bao, N. Wang, M. Chen, and Q. Xu, "Hidden extreme multistability in memristive hyperchaotic system," Chaos, Solitons \& Fractals, vol. 94, pp. 102-111, 2017.

[20] W. Zhou, G. Y. Wang, Y. R. Shen, F. Yuan, and S. M. Yu, "Hidden coexisting attractors in a chaotic system," International Journal of Bifurcation \& Chaos, vol. 28, no. 10, Article ID 1830033, 2018.

[21] M. F. Danca, M. Fečkan, N. V. Kuznetsov, and G. R. Chen, "Complex dynamics, hidden attractors and continuous approximation of a fractional-order hyperchaotic PWC system," Nonlinear Dynamics, vol. 91, no. 4, pp. 2523-2540, 2018.

[22] M. Danca, M. Fečkan, N. V. Kuznetsov, and G. Chen, "Fractional-order PWC systems without zero Lyapunov exponents," Nonlinear Dynamics, vol. 92, no. 3, pp. 1061-1078, 2018.

[23] M. Borah, "On coexistence of fractional-order hidden attractors," Journal of Computational and Nonlinear Dynamics, vol. 13, no. 9, Article ID 090906, 2018.

[24] V. Pham, A. Ouannas, C. Volos, and T. Kapitaniak, "A simple fractional-order chaotic system without equilibrium and its synchronization," AEÜ - International Journal of Electronics and Communications, vol. 86, pp. 69-76, 2018.

[25] N. N. Yang, C. X. Liu, and C. J. Wu, "Modeling and dynamics analysis of the fractional-order Buck-Boost converter in continuous conduction mode," Chinese Physics B, vol. 21, no. 8, Article ID 080503, 2012.

[26] C.-L. Li and L. Wu, "Sliding mode control for synchronization of fractional permanent magnet synchronous motors with finite time," Optik - International Journal for Light and Electron Optics, vol. 127, no. 6, pp. 3329-3332, 2016.

[27] G. C. Zheng, C. X. Liu, and Y. Wang, "Dynamic analysis and finite time synchronization of a fractional-order chaotic system with hidden attractors," Acta Physica Sinica, vol. 67, no. 5, Article ID 050502, 2018.

[28] H. G. Sun, "A new collection of real world applications of fractional calculus in science and engineering," Communications in Nonlinear Science \& Numerical Simulation, vol. 64, pp. 213-231, 2018.

[29] I. Podlubny, Fractional Differential Equations, Academic Press, San Diego, Calif, USA, 1999.

[30] R. Khalil, M. Al Horani, A. Yousef, and M. Sababheh, "A new definition of fractional derivative," Journal of Computational and Applied Mathematics, vol. 264, no. 5, pp. 65-70, 2014.

[31] J. C. Sprott, "Some simple chaotic flows," Physical Review E: Statistical, Nonlinear, and Soft Matter Physics, vol. 50, no. 2, pp. R647-R650, 1994.

[32] A. Charef, H. H. Sun, Y. Tsao, and B. Onaral, "Fractal system as represented by singularity function," IEEE Transactions on Automatic Control, vol. 37, no. 9, pp. 1465-1470, 1992.

[33] C. G. Li, X. F. Liao, and J. B. Yu, "Synchronization of fractional order chaotic systems," Physical Review E, vol. 68, no. 6, Article ID 067203, 2003.
[34] D.-F. Wang, J.-Y. Zhang, and X.-Y. Wang, "Synchronization of uncertain fractional-order chaotic systems with disturbance based on a fractional terminal sliding mode controller," Chinese Physics B, vol. 22, no. 4, Article ID 040507, 2013.

[35] L. G. Zhang and Y. Yan, "Robust synchronization of two different uncertain fractional-order chaotic systems via adaptive sliding mode control," Nonlinear Dynamics, vol. 76, no. 3, pp. 1761-1767, 2014.

[36] X. Wang, A. Ouannas, V. Pham, and H. R. Abdolmohammadi, "A fractional-order form of a system with stable equilibria and its synchronization," Advances in Difference Equations, vol. 2018, no. 1, p. 20, 2018.

[37] Z. C. Wei, A. Akgul, U. E. Kocamaz, I. Moroz, and W. Zhang, "Control, electronic circuit application and fractionalorder analysis of hidden chaotic attractors in the self-exciting homopolar disc dynamo," Chaos, Solitons \& Fractals, vol. 111, pp. 157-168, 2018.

[38] L. D. Zhao, J. B. Hu, Z. H. Bao et al., "A finite-time stable theorem about fractional systems and finite-time synchronizing fractional super chaotic Lorenz systems," Acta Physica Sinica, vol. 60, no. 10, Article ID 100507, 2011. 


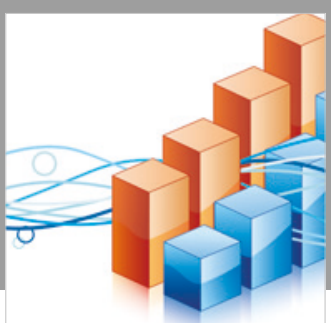

Advances in

Operations Research

\section{-n-m}
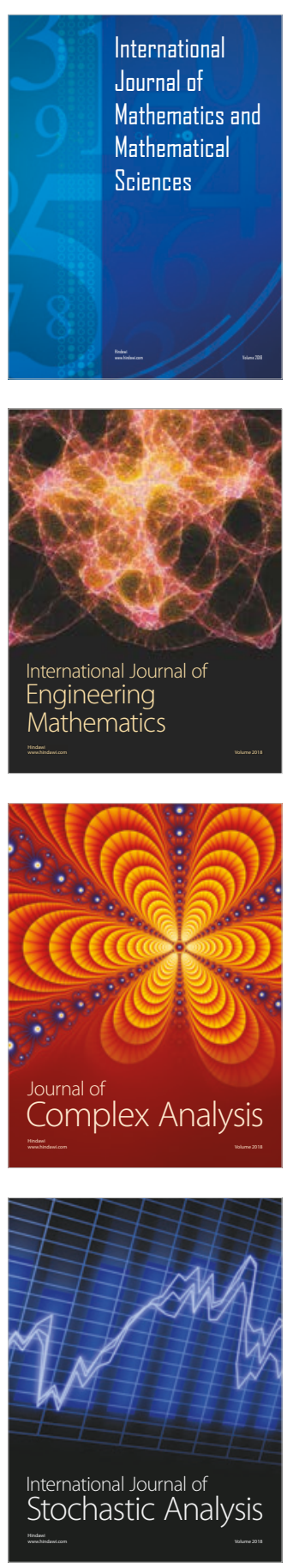
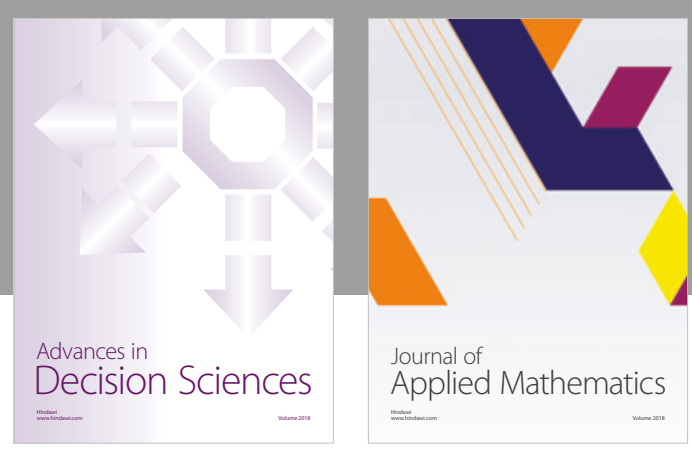

Journal of

Applied Mathematics
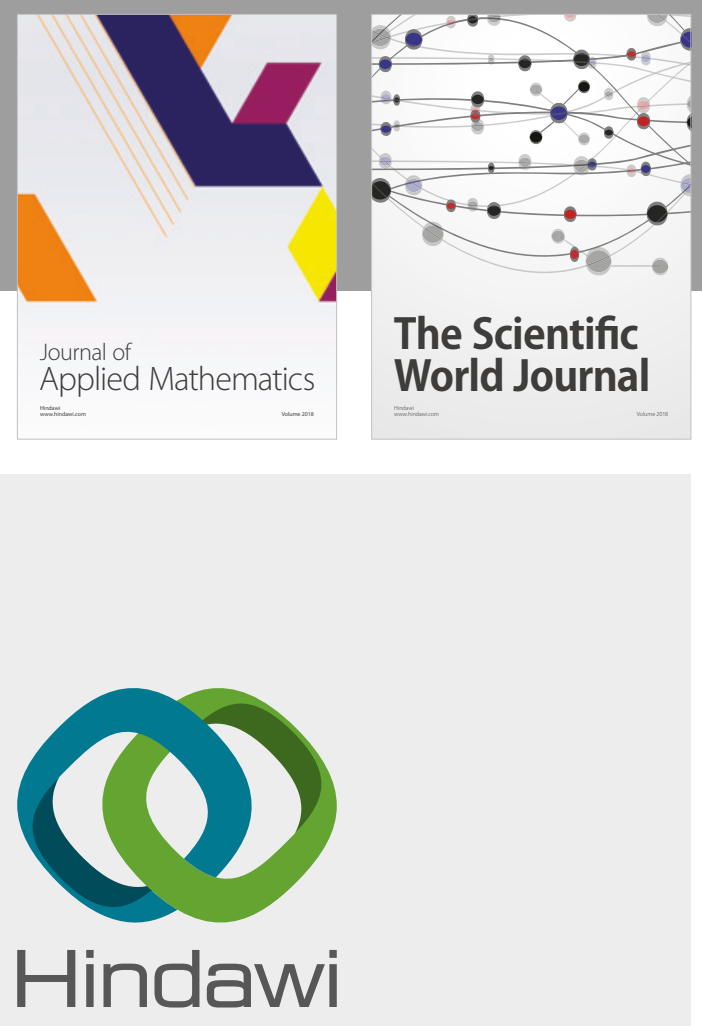

Submit your manuscripts at

www.hindawi.com

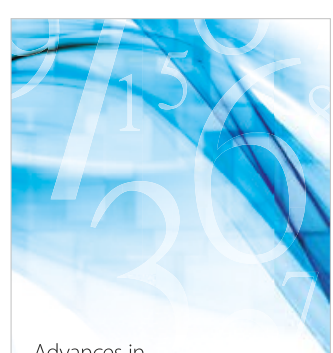

Advances in
Numerical Analysis
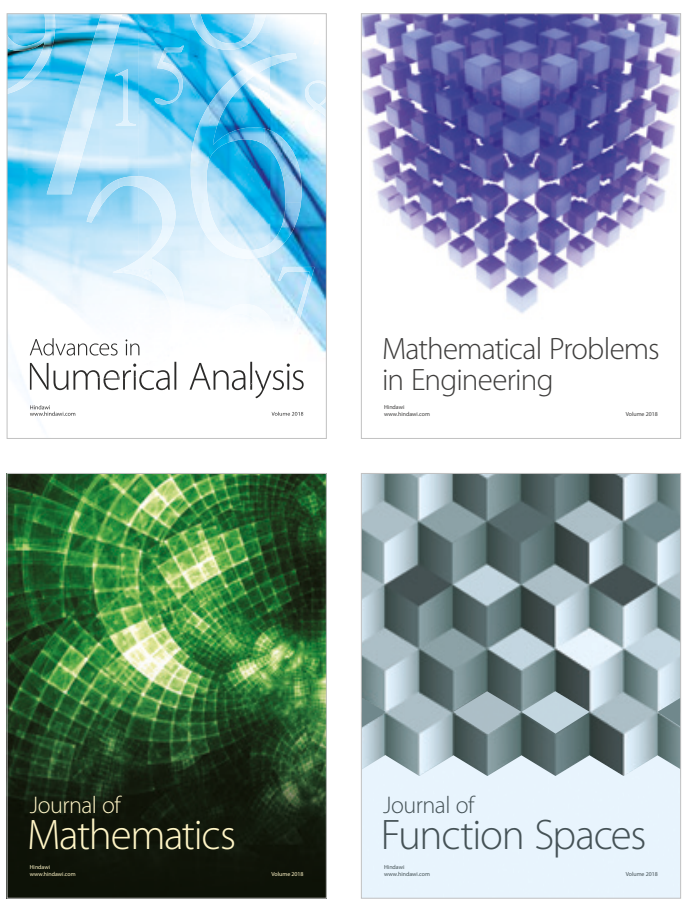

Mathematical Problems in Engineering

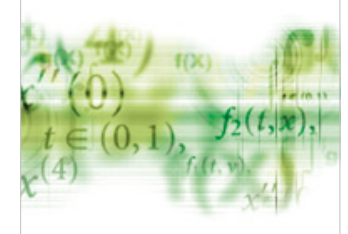

International Journal of

Differential Equations

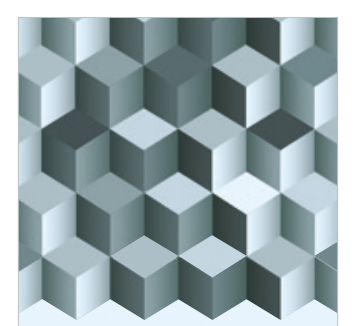

Journal of

Function Spaces

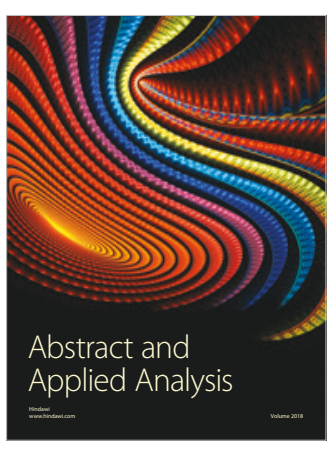

The Scientific

World Journal

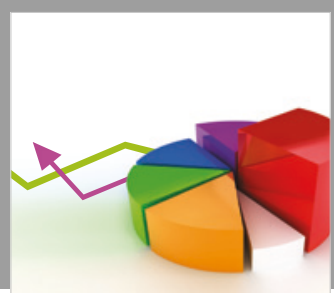

Journal of

Probability and Statistics
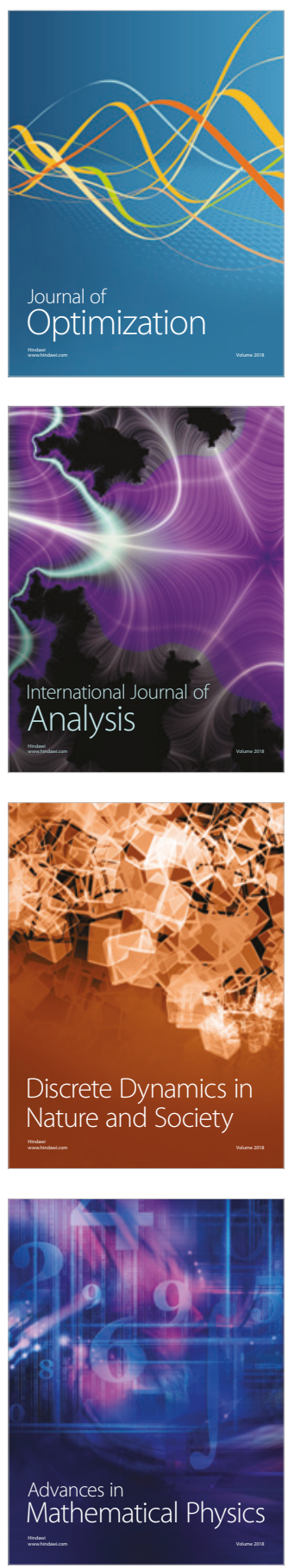